Meta

Journal des traducteurs

Translators' Journal

\title{
L'objectivité dans les langues de spécialité -- cas particulier de la médecine de soins (étude comparée du français et de l'anglais)
}

\section{Dorothy Nakos}

Volume 40, numéro 4, décembre 1995

URI : https://id.erudit.org/iderudit/003692ar

DOI : https://doi.org/10.7202/003692ar

Aller au sommaire du numéro

Éditeur(s)

Les Presses de l'Université de Montréal

ISSN

0026-0452 (imprimé)

1492-1421 (numérique)

Découvrir la revue

Citer cet article

Nakos, D. (1995). L'objectivité dans les langues de spécialité -- cas particulier de la médecine de soins (étude comparée du français et de l'anglais). Meta, 40(4),

701-707. https://doi.org/10.7202/003692ar
Résumé de l'article

L'étude commence par une analyse de la nature de l'objectivité scientifique qui permet d'identifier les éléments pour déterminer les facteurs d'objectivité en médecine de soins (cohérence, précision, justification, neutralité, prudence). On applique ensuite ces facteurs à des ouvrages médicaux fondamentaux écrits en français et en anglais. 
prudence). On applique ensuite (es facteurs d des ouvrages médicaux fondamentaux birits en fransais et en anglais

\begin{abstract}
The article begins with an analysis of the nalure of scientific objectivity. which helps iden rify the elements for determining the factors of objectivity in medical care (coherence. precision. justification, neutrality and (aution). These factors are then applied to fundamental medical writings in French and English
\end{abstract}

\section{INTRODUCTION}

Notre étude vise à montrer les facteurs d'objec tivité qui caractérisent des ouvrages médicaux fondamentaux français et anglais écrits au cours des cinquante demières annés soit par un seul auteur. soit par différents auteurs qui ont chacun rédigé une ou plusicurs parties d'un ouvrage de reférence cherchant l'exhaustivité. Les ouvrages ont été choisis en raison de leur grande diffusion et de la notorieté de leurs auteurs ; ils servent a la formation et à la pratique médicales. Ils ne sont donc pas destinés à des perwonnes travaillant dans le secteur paramédical ni à un public curieux qui lit des texies de vulgarisation scientifique.

L'auteur et le destinataire ont une base cognitive commune (médecine), mais après lecture totale ou partielle de l'ouvrage. le destinataire aura acquis un complément d'information. Pour reprendre les propos. de Kocourek (1982: 19), ala divergence entre l'intention de l'émetteur (de l'auteur) et la compréhension du recepteur (du destinataire) est réduite au minimum». Les textes choisis portent plus précisément sur les maladies : semiologie (ou signes), étiologie (ou cause), description et traitement.

\section{NATURE DE L'OBJECTIVIT}

Avant de déterminer l'objectivité des documents retenus, il nous faut analyser la nature mème de l'objectivite. Selon certains linguistes comme Phal (1968: 11). l'objectivité scientifique "consiste à évincer autant que possible l'experimentateur (sujet humain) pour mettre en vedette l'expérience ou son résultat (objet ou sujet inanime)m. It ajoule : "l'expérimentateur doit toujours être présent salls se montrer, un peu à la manière d'un bon reporter de télévision. On peut dire que dans les textes scientifiques. on ne voit pas l'homme agirn.

Or, dans les textes médicaux didactiques analysés, on ne peut guère parler d' "expérimentateur" ni d expériencew, notions propres aux sciences experimentales dont la medecine experimentale fondée par Claude Bemard. Ce demier, après avoir ohservé un phénomène, procedait a une rontre. epreule experimentale. Dans le cas qui nous intéresse, un médecin praticien observe des signes cliniques chez un patient donne, mais ne les reproduit pas pour arriver à une preuve. La démarche est done différente: le médecin part de l'observation pour 
procéder à l'établissement d'un diagnostic soit sur le champ. soit a l'aide de diverses radiographies ou de tests de laboratoire. L'auteur de textes médicaux pragmatiques observe, réfléchit, interprète el n'expérimente jamais

Lit médecine de soins, contrairement a la recherche médicale expérimentale, sort donc du cadre de l"ubjectivite" ou "objectivation" définie par des linguistes comme Phal que nous venons de mentionner ou Vigner et Martin qui ajoutent que l'objectivité vise a "faire disparaitre tous les procédés servant a exprimer un sentiment ou une appréciation subjectiven (1976: 19). De fatt. la pratique de la médecine demeure un art doublé d'une lechnique (plutôt qu une sciencel visant a dispenser des soins a un patient dans le but de le guérir. L'objectivité ne peut se définir dans ce cas que par rapport al lobjert de crude. cestà-dire la maladie et sat relation avec le patient qui est un humain (ce n'est pas le cals en sciences experimentales) présentant des particularités liées au sexe, à l'âge, a la race, au passé génétique, aux maladies acquises, à l'exercice d'un métier ainsi qu'au contexte sccial et gégraphique.

Déterminer l'objectivité en médecine de soins, c'est donc tenir compte de caracteristiques propres tout en reconnaissant l'apport des découvertes scientifiques, sans lesyuelles la compréhension de la maladie che, l'homme serait impxssible, La communication en médecine de soins s"apparentant davantage, a nos yeux, à une communicalion technique axé vur l'application de connaissances spécifiques a un domaine, nous avons tenu pour acquis l'existence de certains aspects de la comnunication technique déja décrits par Vigner et Mantin (1976: 14 et s49.) et releves dans nos textes. Par exemple: primauté du ficteur référenticl, compréhension entre deux leculeurs situés sur le mème plan, univexité du vocabulaıre et explicité du langage. Toutefois, nous remettons en question d'autres tratits. en particulier la fonction uniquement dénotative du texte et le fait que le discours est nécessairement impersonnel et non marqué.

\section{CRITERES DOBJECTIVITE}

Nous avons identifié des éléments spećifiques servant a déterminer les facteurs d'objectivité en médecine de soins, en l'ik'currence :

\section{- cohérence. \\ - précision. \\ - justification, \\ meutralite. \\ - prudence.}

Chacun de ces criteres sera appliqué aux quatre principaux ouvrages retenus: Mondor ${ }^{\prime}$. Diagnostics urgents - ahblomen. Pasteur Vallery-Radot 2. Pashologie sénérale, (dorénavant PVR dans notre étude), Pentield ${ }^{3}$, The Excituhle Cortex in Comscionus Man et Harrison's Principles of Internal Medicinet. Mondor et Penfield étaient tous deux chirurgiens. I'un de l'atxlomen. l'autre du cerveatu. Le PVR et le Harrison' sont des ouvrages traitant de l'ensemble des maladies, ecrits par divers collaborateurs selon leur spécialité. Les quatre ouvrages sont vendus en librairiet.

\section{Cohérence}

La structure el l'organisation du texte ainsi que la démarche intellectuelle suivie concourent à rendre le discours médical cohérent. En outre, le savoir est plus aisément véhiculé lorsque le cadre est clairement délimités.

Les textes médicaux consultés sont lous structurés de façon rigoureuse : Mondor. PVR, Penfield. Harrison divisent et subdivisent leurs ecrits, apportant tableaux, résumes, listes, dessins et photos a l'appui. Ils utilisent souvent des procédés de mise en relief (caractères grass, italiques, majuscules, soulignement). Mondor, toulefois. ajoutc aux simples procédés typographiques. d'ailleurs fréquents dans la communication technique (exemple fictif : «appuyer sur ON, le voyant rouge s'allume. etc."), le procéde linguistique consistant a rappeler systématiquement un point saillant a l'aide de synagme's de base, par exemple "contracture de la paroi atdominale". Le style de Mondor accumulant énumérations et superlatifs centainement jugess "personnels" par les linguistes contribue aussi a la mise en relief. Par exemple (1965:5):

La contracture vraie de la paroi abdominale. $|\ldots|$ De cous les secours cliniques. il est un des meilleurs : il ne semble pas y avoir, dans toute la pathologic, un symptôme plus utile. plus décisif. plus indispensable, plus riche de sauvetages. On n'en saurait parler avec trop de confiance, de reconnaissance, de foi. C'esl le super-signe de toutes les catastrophes abdominales $[\ldots]$. Ce signe. c'est la contracture des muscles de la paroi abdominale avec ses degrés divers. a la fois d'étendue, de fermeté, de durée $|, .$.

L'auteur poursuit en reprenant régulièrement le syntagme de base

Mondor demeure l'auteur yui foumit sans doute une des descriptions les plus approfondies des signes de la maladic. Sa particularité est de présenter une image englobante, de revenir sur un signe essentiel. puis d'expliciter dans les moindres détails tous les aspects liés a la maladie décrite. Son texte est également organisé de façon logicue, par exemple (196.5:5):

Contracture de la paroi abdominale

- signification physiologique :

- conditions cliniques (avec photos a l'appuil :

- risques d'erreurs.

De plus, il introxluit des tableaux comparatifs permettant aux médecins d'établir un bon diagnostic: il distingue sur deux colonnes "la lession abdominale" et "la lésion thoracique" en indiquant chaque fois comment identifier les signes se rappontant aux deux étages de lessions (196.5:37-38). 
PVR adopte un modele qui s'apparente à celui de Mondor dans la mesure où le lexte s'inscrit dans le ciadre général de la physiologie. Exemple (1963:233):

Physiologie - sémiologie [ Ire partie]

Physiologie de la digestion IPVR en donne une definition courne et précise : «acte physiologique qui prepare les aliments en vue de leur absorption dans l'intestin grêle et de leur métabolisme».)

I. Ia cavité buccale

II. l'estomac letc.]

Dans chaque section, on retrouve les sous-titres "phénomènes mécaniques *. puis uphénomènes cliniquesm s'il y a lieu.

Il semble bien que la démarche intellectuelle des deux auteurs français soit d'avoir toujours comme point de départ la physiologié; par exemple, si nous reprenons lexemple du PVR, la physiologic de la digestion traitant des mecanismes de la digestion allant de la houche à l'anus; viennent ensuite la sémiologie ou signes liés a la pathologie de l'appareil digestif dans l'exemple choisi, la physiopathologie, c'esta-dire l'étude des modifications des grandes fonctions de l'organisme au cours des maladies. la pathologie et enfin le traitement une fois le diagnostic etabli.

Pour ce qui est des ouvrages anglais, l'acces à l'information est trds directe, sans suivre le raisonnement allant du général au particulier. fondé sur la physiologie, propre aux ecrits français. Contrairement au cadre large des auteurs français, le cadre est ici intentionnellement rétréti. Le découpage le plus net se retrouve centainement dans l'ceuvre de Penfield qui introduit en plus un plan au début de chaque chapitre. Par exemple (chapitre VII rédigé par Penfield dans Ponfield el Roherts 1959) :

Mapping the Speech Area

A. Forbidden territory

B. Procedures in opering room

1. Stimulator and sfimulatory electrode

2. Routine of cortical mapping

3 Acrivation and arrest

C. Exploration of Spech Territory

D. Case example

Par ailleurs. Penfield fait un plus grand usage de dessins que les autres, ce qui peut étre perçu comme l'indice d'un esprit analytique. le dessin analysant beaucoup mieux que ne le fait la photo qui synthétise. Ses ecrits sont aussi beaucoup plus concis.

Dans le Harrison. les éléments d'information sont juxtaposés : par exemple, "Biological considerations», "Clinical considerations" si bien que le lecteur trouve facilement l'information selon la rubrique qui l'intéresse. L'information figurant dans le Harrison est "prédigérée" en quelque sorte car elle présenie demblec une vision synthétique de la maladie. Mondor. PVR. Penfield analysent la maladie arant d'en faire la synthese. Le Harrison est un ouvrage synoptique adapté a la pratique médicale de plus en plus façonnéc sur le modele américain. ce qui explique sans doute sa très grande diffusion et le fait qu'il a été traduit en sept langues. En outre. Ie Hartison met l'accent sur des maladies "vedettes": maladies génétiques, cancer, deficience immunitaire.

On pourrait croire que les textes qui donnent l'acces le plus direct à l'information seraient les plus objectifs de tous : d'ailleurs la communication ^directe de l'information serait un facteur caractéristique de toute communication technique selon Vigner et Martin (1976: 17). Or ie Harrison qui juxtapose des élements d'information pour arriver à une synthèse «prédigécée n'incite pas vraiment le lecteur a reflechir en tirant ses propres conclusions sur la maladie. En ce sens, le textes du Harrison. ce qui semble paradoxal, seraient moins objectifs que ceux de Penfield, Mondor et PVR.

\section{Precision}

La précision du vocabulaire est un trait des langues de spécialite (lsp). Rappelons ici qu'une isp esi une langue permettant à des auteurs de méme niveau de transmetre un savoir donne de la Connaissance ou les procédés d'application d'une connaissance a des fins pratiques. Le vocabulaire de la médecine est précis et en perpétuel renouveau en fonction des decouvertes medicales (par exemple. le terme wempyeme" est devenu désuet parce que la thérapeutique par les antibiotiques a supprimé la formation de collections purulentes dans les plèvres: en outre. Ies nouvelles techniques de l'imagerie médicale ont donné lieu a la création de toute une série de termes). Une étude antéricure (Nakos 1989: 352-3.59) nous a prouvé que la présence de syntagmes terminologiques complexes etait encore plus marquee en médecine que dans d'autres Isp. le syntagme de base étant généralement augmenté au fur el à mesure que la médecine progresse. De plus, dans les ouvrages medicaux consultes, on ne retrouve pas de synonymes terminologiques ce qui, dans le cas des Isp, est indice de rigueur. Signalons, loutefois, la présence de termes latins, par exemple "fetor hepaticus" (oxdeur aromatique de l'haleine) (PVR 1963: 484) et de termes anglais répandus que le français juge parfois utile d'ajouter, par exemple oflapping tremor" (PVR 1963: 484).

Par ailleurs. I'utilisation d'un vocahulaire imagr concourt à rendre le texte précis, surtout pour décrirc les signes en français, par exemple "groseilles écrasees", wteinte chocolat", "ventre de bois" pour la contracture abdominale. Nous ne tenons pas compte ici des images perdues, empruntées, comme acirrhose" qui signifie "roux" en grec. Ie foie malade" ćtant roux d'après l'observation que Laěnnec en avait faite. Le vocabulaire concrel du Harrison est particulièrement fertile : wapple-core lesion" (1991 II : 1221) unutcracker oesophagus" (1981 11 : 1225). *corkscrew oesophagus" (1991 II : 1225), "succussion splasho (1991 11: 1234), «leathery friction.. aleather bottle appearance" (1991 II : 1249), "coffece grounds appearance" (199) II : 261-262), "stools are tarry (sticky) $\%$ [...] "clay-colored $*$ (199] II : 26K). 
"cross-beta pattern, pleated sheet" (1991 II : 1418), "cobblestone arrangement of liver cells" (199) II : 1337). Les métaphores utilisées s'appliquent a la clinique ou à la radiologie, mais non aux techniques de laboratoire. On voit que la précision peut être atteinte par l'emploi de métaphores ancrées dans l'usage médical. ce qui n'est pas évident pour l'ensemble des Isp.

Mondor, liltéraire en plus d'ètre chirurgien. utilise aussi les images à des fins stvlistiques pour rendre son récit vivant. On est loin de l'impersonnalite généralement souhaitée dans les Isp. Les collabo. rateurs de Mondor comme Henri Benard employaient également des effets stylistiques de ce genre. Par exemple (19.52: 224):

On compare volontiers le foic a une vaste raffinerie des matières premieres, double d'un entrepôt de produits nutritifis et d'un filtre a mailles etroites, branché en dérivation sur le tuhe digestif.

\section{Justification}

Toute lsp comporte une justification des donnees sous forme de reférences, citations, chiffres, stalistiques entre autres. Selon Kocourek (1982: 19). la référence et la citation sont la base du edialogue scientifique simulé, entre auleur et lecteur. Dans les lextes médicaux retenus, lat justification ste situe a des niveaux differents. Les auteurs citent tous leurs sources quand 11 sagll de réfórence's immédiates. Le Harrison s'en tient d'ailleurs uniquement à ce type de références. On y trouve une vision ponctuelle des faits. C"est egalement l'ouvrage qui présente le plus de données statistiques. Par excmple (1991 II : 1249):

It is estumated that the diagnosis was made in 10.160) Americans in 1989. leading to 9410) deaths $|\ldots| .|\ldots|$ the disease fappears $\mid[\ldots \mid$ most frequently after $50|\ldots|,|\ldots|$ \&0 to 90 percent of |... | cases are telieved attribuable to excess consumption of alcohol (...). Approximately 1.5 percent of exophageal cancer occurs in the upper third of the esophagus $[\ldots], 50$ percent in the middle third |... I. I cess than 5 percent of patients are alive 5 years after the initial diagnosis $\mid \ldots l$. Resection $(\ldots \mid$ is feasible in only 40 percent of cases |... I. I.ess than 20 percent of patients who survive $|. .$.$| resection can be expected to be alive$ after 5 years. The therapeutic outcome following the admonistration of primaty radiation $[\ldots]$ (15.5 60 60) Gy (5.5(x) (o $6(M)$ Rad)) is not dissimilar to that of radical vargery

Ie fatt, le Harrism, comparativement aux autres ouvrages, accorde une importance demesuré aux statistiques et aux chiffres, soit pour déterminer un groupe cible de patients, comme dans lextrait cite. soit pour déterminer le dosage de médicaments : par exemple (1991 11: 477): "prednisone (40) (1) $60 \mathrm{mg}$ dallyl is recommended".
Le recours systématique à des statistiques pourrait être considéré comme un élément déterminant de l'objectivité. Encore faudrait-il savoir comment elles sont établies et si l'interprétation qu'en fait l'auteur est necessairement exacte.

Par ailleurs, Mondor, PVR et Penfield, grands "patroms" de la médecine. ne se sentent pas toujours obliges de citer leurs sources, du moins dans le cas de travaux anterieurs, éloignes dans le temps. Mondor, qui a de vastes connaissances de la médecine, passe rapidement sur les travaux connus de l'ensemble des medecins pour mettre en lumiere les étapes marquantes d"une découverte. Fen cela, il se départit de la maniere de faire des Americains qui placent sur le même plan toutes les donnés, toujours rapproxhées dans le temps. Mondor remonte le fil des ans pour montrer de façon claire et vivante les faits saillants d'unc découverte. Par exemple (196.5: 19):

|... I la première étude de quelque importance que j'ai trouvée est celle d'Fbstein qui, en Allemigne. en IX8.5 [etc.]

Il est volontairement vague quand il juge que la date ou les statistiques sont sans imporance. Voici un exemple typique (196.5: 19) :

Si les statistiques [il ne les donne pas] [...] sont

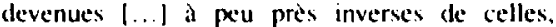
macabres. d'il y a trente à quarante ans lapproximatifl. il taul considerer avec lecene, qui le disait a la Societe de Chirurgie de Paris il y a peu de temps |on ne sait pas trop quand $|\ldots|$ que tous les progrès $[\ldots \mid$ reposent $|\ldots|$ sur la contracture vraie de la paroi abdominale.

PVR et Pentield présentent, tout conme Mondor, des éléments historiques en étahlissant une hiérarchie entre les faits connus. Mondor. PVR et Penficld paraitraient moins objectifs selon les normes des lsp. mais, de fait, ils se fondent sur letur apérience plutot que sur de's donnótes chiffrés on une bibliographes chanesfre qui ont surtout pour rôle de rassurer le lecteur (et l'auteur) yuant au séricux des travaux.

\section{Neutralité}

1.e textes medicaux s'evaluent egalement en fonction 1 "de la présence ou de l'effacement de l'auteur médecin et $2^{\circ}$ de la perception du patient par le métecin.

Ches Mondor. le medecin occupe une place prépondérante. Le "jo" sujet est omniprésent dans limerprétation des fats rlinique's toujours étofté : mais la descraption de's signes observe's. comme nous lavons deja constatte, demeure objective. Exemple (1965: 78$)$

Une douleur atroxe et vive diffuséc avec traînée a gauche $|\ldots|$. Peu de vomissements $|.$.$| . une$ diarrhé fétide, choléforme. Ine température peu elevee : $38^{\circ}$ |... Mais un pouls a 140, faible, 
fuyant. Le faciès est plombe. tire, gris, hebette, anxicux $[\ldots]$

Il relate, en outre, bon nombre d'anecdotes personnelles prenant l'allure de romans policiers. Exemples (1969: 79 el 8.3) :

Un médecin me téléphone, une nuit [...]. C'est. dit-il, une appendicite. La croyez-vous urgente? Pour gagner du temps, je fais transporter la |... malade pendanı la nuit $|. .$.$| Je l'examine : 38^{\circ} 4$. pouls a 120 . contracture généralisée $(\ldots)$ Je l'opere enfin $|\ldots|$

J'ai vu la mort survenir $(\ldots)$ je vois l'enfant à 16 heures, c'est-a-dire vingt-quatre heures apres le debut. $\mid$... $\mid$ j'opere a 18 heures.

Mondor prend aussi un ton dramatique quand il renforce les substantifs par des qualificatifs d'intensité : «terrible surprise !». "douleur atroce", «opposition malheureuse". "remarque amere". Il est Également emphatique : «la Péritonite aiguè", avec un grand “P». Il pontifie el sunout. comme on l'a déja mentionné au sujet des procédés de mise en relief. il insiste et revient régulièrement au signe principal, un peu a la maniere d'un theme repris dans une symphonie. Son ceuvre contient des envolees lyriques. el pourtant elle demeure pragmatique. Elle est complète : la forme che: lui renforce le fond.

Mondor présente aussi les erreurs d'interpretation de ses collegues qu'il appelle "fausses interpretations». Comme on peut le constater, on s'ecane considérablement du modzle soi-disant impersonnel des Isp. Kocourek est l'un des rares linguistes a avoir nuancé son propos au sujet de l'impersonnalité (1982: 19): "Contrairement a ce que l'on croit, il n'est pas impossible de montrer que lémothivité (affectivité) participe a la communication de spécialite». Néanmoins, il ajoute que "la langue technique ct scientifique vise l'impersonnalité.

L'écriture de PVR et de Penfield est dense et pondérte : les formules du genre ad'une part - d'autre part". "premierement - deuxiemement» abondent. Contrairement a ce qu'avance Phal (1968: 11 et sqq.). le passif typique des Isp est assez rare chez. PVR qui privilégie les toumures actives: "les diverticules realisent" (196.3:431); al estomac retient les aliments |... J. les malaxe $[\ldots]$, les dilue $[\ldots \mid$ (1963:235). Le texic, elégant. évite également les chamières comme "en outre", "par ailleurs", senfin", particulières d la démarche logique du français qui établit ainsi un lien entre les parties du discours.

En ce qui a trait au patient. comme on peut s'y attendre. Mondor le personnalise. il manifeste souvent de l'affection à son égard et sa joie quand il arrive a le guérir (ale petit malade», uun perit gasçon de huit answ. etc.). C'est le seul à monirer de l'émotion. Penfield particularise ses patients et les fait parler (1958: 30, 29).
The patient M.M. [...] was a good witness, selfcritical. understanding and tolerant: she said, "No". Then she said "Yes", I suddenly cannot hear.

PVR, quant a lui, genéralise : "chez la femme" "chez le nouveau-ne" etc. ce qui est maintenant la norme des articles français. Le patient est perçu selon l'age et le sexe. Il est de plus en plus anonyme. Le Harrison trace un portrait type. par exemple «American male, black, under 50), smokes, (etc.|m. Le malade devient entité neutre.

\section{Prudence}

D'une façon générale. le ton est affirmatif quand le traitement est connu et utilise par la pluparn des médecins, ce qui semble aller de soi, et nuancé quand il n'existe qu' un traitement palliatif. Ainsi «should be (used, etc.)\% accompagne le plus souvent les indications therapeutiques alors que dans les autres cas. la prudence simpose et se reflète dans le choix des verbes: "may be (viewed, initiated, recognized)". "may occur", scan be (expected, believed)", "suggesting*. "it is possible», «appear to be". En outre, les adjectifs d'intensité approximatifs comme $*$ substantial*, "marked", "considerable". "moderate" ajoutent de la prudence au texte.

Le ton est aussi affirmatif quand il s'agit d'une intervention pratiquee sur un cadavre : le cadavre esl fixé par formol et la dissection n'entraîne aucune complication qui aurait des effets juridiques. Par contre. dans le cas d'un être vivant, le ton devient beaucoup plus nuancé. En effet. l'intervention chirurgicale esl difficile sur un tissu vivant et non fixé : en outre, les medecins pourraient se tromper et subir des sanctions penales. Cela dit, le Harrison demeure le plus prudent des ouvrages consultés. Selon nous. la prudence marquerait l'objectivite dans la mesure où elle expose une lacune dans le résultat de l'observation. Flle est indice d'honnéteté et partant, de vérité.

\section{CONCLUSION}

Les fondements de l'objectivite de toute Isp demeurent, selon nous. la référentialité et l'absence de déformation dans les faits rapportés par un auteur qui s'adresse a un lecteur de méme niveau, ce qui se retrouve dans les textes médicaux retenus.

Cohérence, precision, justification. neutralite. prudence, telles que nous les avons déterminées. sont les élements spécifiques de l'objectıvilé médicale. reprétsentés de façons différentes dans les textes choisis. Le discours marqué, personnel, connoté, relevé particulièrement chez Mondor, sent a convaincre davantage le lecteur en retenant son attention. Le style riche. varie de Mondor et travaille de PVR et Penfield ne sont pas indice de pauvreté intellectuelle! Mondor et Penfield ont marque leur epoque: leurs ecrits continuent a circuler car ils ont produit de véritables "Bibles" médicales. La forme complète le fond pour produire un document limpide. structuré dans lequel les points 
essentiels sont nettement dégagés. Le fil conducteur y est toujours présent.

Malheureusement, la liberté de l'expression médicale est sérieusement atteinte : des auteurs français comme Hamburger $(1982: 180)$ indiquent même aux médecins un modèle unique à suivre pour écrire des articles. en six étapes (1. Titre et liste des auteurs. 2. Introduction. 3. Matériel et méthodes. 4. Résultats, 5. Discussion, 6. Résumé) en précisant que les mots-clés doivent toujours figurer dans le titre afin de faciliter le classement documentaire et, sans doute maintenant, l'insertion dans les banques de donnees bibliographiques. Le modèle américain du Harrison s'étend et gruge les écrits médicaux. Le livre est au service de la profession. La simple juxtaposition des faits observes, sans réflexion, et le morcèlement de l'information qui s'ensuit, semble la norme. Elle a l'avantage, selon les experts, de faciliter l'automatisation des données.

Le médecin humaniste devient un technicien. L'efficacité, fondée sur la production et la diffusion, est désormais la règle d'or. Au nom de cette efficacité. on produit des textes français calqués sur l'américain sur le plan de la pensée et de la structure. D'ailleurs, on a de plus en plus tendance à emprunter l'anglais comme langue de communication dans les ecrits médicaux français: depuis 1989, les trois revues specialisées des Annales de' I'Institur Pasterur (immunologie, virologie et microbiologie) sont devenues anglaises. Seuls les articles rédigés en anglais y sont acceptés. Il y a de quoi reflechir! Le français médical est en train de vendre son âme à l'américain.

\section{Notes}

1. Henri Mondor était a la fois chirurgien de l'abdomen et littéraire (a écrit sur Mallarmé. Valéry, Alain). Il est décédé en 1962. Diagmostics urge'nts - abdeme'n, wojours vendu, a êté rédité pour la neuvième fois. Mondor était membre de l'Académie de médecine, de l'Académie française el de l'Académie des sciences.

2. Louis Pasteur Vallery-Radot était spécialiste des maladies des reins el des altergies. C'etait le petit-fils de louss Pasteur dont il publia les Gienres complies et lat correspondance. Il a Également écrit un livre sur Pasteur (Pasteur. ce't incommol. Il ess décédé en 1970. Pathologie geinérale est l'ouvre de treise collaborateurs. (et ouvrage a ensuite ele divisé en fascicules. Ie fascicule sur la gynécologie et la repreduction et celui sur la néphrologic fou maladies des reins) et l'urologıe, rédités pour la deuxième et la troisième fors respectivement (1975 et 1980) se trouvent sur le marche.

3. Wilder Penfield, neurologiste canadien décédé en 1976, ent celebre pour ses recherches sur le trattement chirurgical de l'eppilepsic. Ses travaux demeurent d'actualite.

4. Harrison's principles of Internal Medicine regroupe des chapitres rédigés par 317 collabo). rateurs soigneusement choisis. Cet ouvrage a été traduit en sept langues depuis 1988 .

5. En particulier, les sections traitant des maladies lices à l'appareil digestif.

6. Mondor, Pasteur Vallery-Radot et Penfield figurent tous dans les dictionnaires de noms propres dont le Le' Petir Rohert 2.

\section{REFERENCES}

A. Outrages linguistrque's

DESCAMPS, Jean-Luc et André PHAL (1968); “La recherche linguistique au service de l'enseignement des langues de spécialitém. Le français dans le monde, $n^{\circ}$ 61, décembre. $8^{e}$ anné, pp. 12-19.

DIRCKX. John H. (1976): The Language of Medicine. Its Evolusion. Structure and Dynamics, New York. Harper \& Row, 171 p.

HAMBURGER, Jean (1982): Introduction au langage de la médecine. Paris, Flammarion. Médecine / Sciences, $202 \mathrm{p}$.

KOCOUREK, Rostislav (1982): La langue frangaise de la technique er de la science. Paris, La documentation française, $259 \mathrm{p}$.

KOCOUREK, Rostislav (1991): La langue francaise de la sechnique et de la science. Paris, La documentation française. $2 c$ ed. $327 \mathrm{p}$.

NAKOS, Dorothy (1989) : "Etude comparee des modes de formation de lexies complexes dans deux domaines différents». Mesa, vol. 34, $n^{\circ}$ 3. septembre. Actes du colloque des terminologies specialisées: Approches quantitative el logico-sémantique. pp. $352-354$.

PHAL., André (196K) : "De la langue quotidienne a la langue des sciences et des techniques". Le

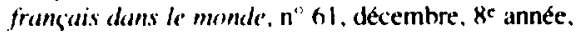
pp. 7-12

SAGiE. Yvan et David DUIN(iWORTH (1980): English Special Languages. Principles and Practice in Sicience and Techmology, Wieshaden. Oscar Brandstetter, $368 \mathrm{p}$.

VIGiNER, Gerard el Alix MARTIN (1976): le francsis lertmique'. Paris, Librairies Hachette et Larousse. coll. «le français dans le monde». $111 \mathrm{p}$.

B. Ourrages médic aus

Annale's de' l'Instinut Pasterar / Actualités (1990) : vol. 1. n" 1-2-3-4, Paris, Editions scientifiques Elsevier. (ieorges $\mathrm{N}$. (ohen rédacteur en chef.

MONIORR. Henri (196.5): Diagnensirs argents abdumen, Paris, Masson et Cie, $9^{\circ}$ ed., $1119 \mathrm{p}$.

MONDOR. Henri. DHEBRÉ, Robert el coll. (19.52): Sermme de médecine comermporsine. Nice. Les édittons médicales La Diane Française, 444 p.

PASTEUR VALLLERY-RADOT, Louis, HAMBURGER. Jcan. LHERMITE. François, CONTE, Michel, RYCKEWAERT. Antoine et coll. (1963) : Pathologes seingrale. Paris, Edition médicale Flammarion, 5" ed. lost p. (vol. "reins, appareil digestif, maladies allergiques, os et articulations. muscle-avitaminose, maladies generales diverses"). 
PENFIELD, Wilder (1958): The Excitable Cortex in Conscious Man, Liverpool. University Press, coll. "The Sherrington Lectures $m$, vol. S. 42 p.

PENFIELD. Wilder et Lamor ROBERTS (1959): Spech and Brain Mechanisms. New Jersey, Princeton University Press, $286 \mathrm{p}$.

SOURNIA. Jean-('harles (1991): Histoire de la médecine el des médecins, Paris, Larousse, 585 p.

WILSON, Gene D. et Editors (1991): Harrison's Principles of Internal Medicine. Montréal. McGraw-Hill. $12^{e}$ ed.. 2 vol.

DOROTHY NAKOS Universite Laval, Québec, Canada 